\title{
Perioperative therapy in HER2+ patients
}

\author{
R. P. A'Hern ${ }^{\mathrm{a}}$, N. J. Bundred ${ }^{\mathrm{b}}$, on behalf of the EPHOS-B Trial Management Group \\ ${ }^{a}$ Clinical Trials and Statistics Unit, Section of Clinical Trials, The Institute of Cancer Research, Sutton, UK; \\ ${ }^{b}$ University of Manchester/University Hospital Education and Research Centre, Manchester, UK.
}

\begin{abstract}
Use of perioperative anti-HER2 therapy presents an opportunity to use a therapy which has been shown to be effective in the adjuvant setting early in the course of a patient's primary treatment, to assess tumour response in vivo using biomarkers and to potentially improve outcome by suppressing the growth stimulus to cancer cells that is thought to occur due to surgery. Biomarkers measured both before and after a short period of preoperative therapy may offer important predictive and prognostic information that can guide future therapy and follow-up. However, the safety and value of perioperative therapy in this context has not been established and its investigation should therefore be conducted within the context of a randomised, controlled trial. Such a trial, EPHOS-B, will shortly commence in UK alongside POETIC, a parallel trial of perioperative therapy in hormone receptor positive postmenopausal breast cancer.
\end{abstract}

Keywords: HER2+; Perioperative therapy; Primary breast cancer

\section{Introduction}

Perioperative therapy offers a number of potential benefits including the ability to counteract the possibly negative effects of surgery, the ability to give effective therapy early and a gain in prognostic and predictive information. There is evidence [1] that surgery stimulates the growth of breast cancer cells and causes the release of cancer cells that may then be able to form metastases; perioperative therapy may inhibit these two processes. Secondly, trastuzumab is an effective therapy, which is often not given until after chemotherapy but could be used sooner, if given in the perioperative setting. Presurgical therapy also offers the opportunity to measure response

Correspondence to: Roger P. A'Hern, Clinical Trials and Statistics Unit, Section of Clinical Trials, The Institute of Cancer Research, Sutton, SM2 5NG, UK. E-mail: Roger.Ahern@icr.ac.uk; Tel: 0208722 4056; Fax: 020 87707876

Received: $22 / 11 / 08$

Accepted: 08/05/09

First published online 18/06/09

BCO/599/2008/FO to treatment in vivo, giving information on the effect of treatment on biomarkers such as proliferation, apoptosis and gene expression, yielding potentially valuable prognostic information. Short-term presurgical models have been advocated to evaluate endocrine resistance mechanisms [2] and are more generally applicable to other interventions. However, if they are used for this purpose, it is important to show that use of intervention does not disadvantage individual patients. Large randomised trials such as that carried out with neoadjuvant cytotoxic chemotherapy by National Surgical Adjuvant Breast and Bowel Project in trial B18 [3] are needed to address such issues. Resistance mechanisms can also be investigated by associating changes in biomarkers, which are responsive to the intervention, such as Ki67 or apoptosis, with the expression of putative determinants of resistance. For example, little change in Ki67 would be expected in ER (oestrogen receptor) negative patients in response to presurgical treatment with an aromatase inhibitor. The biology of novel treatments can therefore be studied using this method; a current example examines the 
biological effect of lapatinib in unselected patients in a presurgical randomised controlled trial known by the acronym MAPLE [4].

A large, randomised, controlled trial of perioperative anti-HER2 therapy supported by Cancer Research UK is planned within the United Kingdom. EPHOS-B is a phase III, multicentred, three-arm, randomised controlled trial of perioperative antiHER2 therapy in which HER2+ patients will be allocated in a $1: 2: 2$ ratio to control, perioperative trastuzumab or perioperative lapatinib. Perioperative treatment will consist of 11 days ( \pm 1 day) preoperative treatment and a short course of postoperative treatment. It is anticipated that patients will receive further trastuzumab treatment after adjuvant chemotherapy. Centres may choose at their discretion whether to take into account that some patients have already received serveral weeks of peri-operative anti-HER2 therapy when prescribing their standard adjuvant trastuzumab. This is a trial with biological endpoints, undertaken to examine whether an 11-day treatment period is able to produce biological changes compatible with a treatment effect. EPHOS-B will determine the definitive design of the following larger clinical study, EPHOS-C, which will assess the therapeutic value of perioperative therapy. EPHOS is being coordinated by The Institute of Cancer Research Clinical Trials and Statistics Unit (ICR-CTSU) in collaboration with The University of Manchester. POETIC [5], a parallel trial of 2 weeks of aromatase inhibitor therapy in hormone receptor positive postmenopausal patients, is also being coordinated by ICR-CTSU. Evidence of the biological efficacy of short-term preoperative aromatase inhibitor treatment already exists; POETIC has therefore been initiated as a 4000-patient trial with both efficacy and the predictive/prognostic effects of biomarker changes as endpoints.

The importance of the effect of surgery on breast cancer cells is still a matter for debate. Evidence that surgery stimulates the growth of breast cancer cells has been provided by Tagliabue et al. [1] who examined breast cancer cell proliferation (MIB-1) in paired primary and re-excision specimens removed within 48 days of surgery. They found that the latter showed a significant increase in proliferation if they were HER2 + but not if they were HER2 - negative (Fig. 1). Forty-three tumours, comprising 86 samples, were retrieved from 32 patients, 24 of which were carcinoma in-situ and 19 invasive cancer. Overall, the median percentage change in MIB-1 was -2 (95\% Cl: -10 to 2$)$ in HER2 - tumours and 8 (95\% Cl: 4 to 16$)$ in HER2 + tumours, $P=0.0007$. In HER2 + tumours, the mean number of MIB-1positive cells was found to increase from 16.2\% (SD

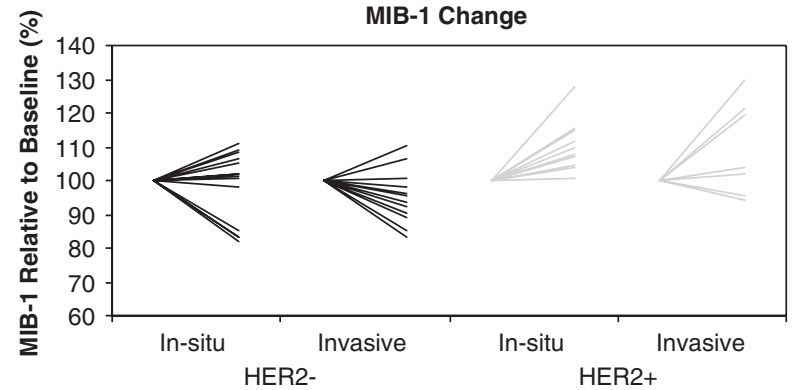

Figure 1.

Changes in MIB-1 in 43 tumours from 32 patients obtained following re-excision for residual disease. The tumours were removed within 48 days of surgery. Adapted from Tagliabue et al., each line shows the MIB-1 change from baseline of a single patient.

11.7 ) to $26.3 \%$ (13.6), an increase in proliferation of approximately $50 \%$. In addition, the difference between HER2 - and HER2 + tumours was also individually significant in carcinoma in-situ and invasive cancer patients $(P=0.01$ and $P=0.03$ respectively). Fisher et al. [6,7] demonstrated a similar phenomenon in a mouse model; implanted human cell proliferation was found to follow tumour removal, this proliferation was also prevented by preoperative therapy.

The ability of disseminated cancer cells to establish metastases is also a matter of controversy. Choy and McCulloch [8] found that although tumour-cell shedding occurs during surgery on primary breast cancers, after surgery the released cells are cleared from the circulation. It has also been predicted by some authors that only one in every 10000 cells succeeds in forming a metastasis [9]. Circulating tumour cells have to settle at a distant vascular bed, make their way through the vessel wall into the target organ parenchyma and then be able to grow. Whilst this is happening, the cells must evade the host immunological response and be able to survive possibly adverse metabolic conditions. Spread of tumours through the bloodstream is hence likely to be an inefficient process and most circulating cells may not possess the characteristics to establish metastatic disease, or are overcome by adverse host factors [10].

\section{Perioperative anti-HER2 therapy}

There are a number of outstanding questions regarding the use of anti-HER2 therapies in addition to the value of perioperative therapy. Little is known about the optimum use of lapatinib but more evidence exists about the use of trastuzumab from clinical trials. One issue concerning the use of trastuzumab that has not been resolved is the 
optimum duration of therapy. It was observed in the HERA trial [11], for example, that 12 months of trastuzumab reduces the increased hazard rate seen in the untreated group during the first year, but has less effect beyond this point. A trial comparing observation with trastuzumab for 9 weeks found a $60 \%$ reduction in the event rate over a median follow-up of 36 months [12]. The FinHer trial included 232 early breast cancer patients and a hazard ratio favouring trastuzumab of $0.42(95 \% \mathrm{Cl}: 0.21$ to $0.83 ; P=0.01$ ) was observed for recurrence or death in this study. A short duration perioperative course of trastuzumab may, therefore, offer a proportion of this benefit early in the primary treatment phase. There is also evidence $[13,14]$ that preoperative trastuzumab is effective in improving pathological complete response rates. A study designed to determine whether the addition of trastuzumab to neoadjuvant chemotherapy could increase pathologic complete response $(\mathrm{pCR})$ rates in patients with HER2 + disease was terminated early due to an indication of a large benefit for the trastuzumab group [15]. Patients were randomised to either four cycles of paclitaxel followed by four cycles of fluorouracil, epirubicin and cyclophosphamide or to the same chemotherapy with concurrent weekly trastuzumab for 24 weeks. The intended trial size was 164 patients but it was stopped when 42 patients had been entered, of which $26 \%$ in the chemotherapy arm achieved pCR compared with $65.2 \%$ in the trastuzumab plus chemotherapy arm $(P=0.016)$. A further unresolved issue regarding the use of trastuzumab is whether it should be started concurrently with taxane chemotherapy (NSABP B-31, NCCTG N831 [16]) or is better started after completion of chemotherapy (HERA [11]).

The hypothesis that systemic therapy should be given preoperatively, was first tested in a large randomised trial by the National Surgical Adjuvant Breast and Bowel Project in trial B18 [3], which evaluated the role of preoperative chemotherapy $(A C \times 4)$ compared with the same duration of chemotherapy given after surgery. Trials of short-duration chemotherapy given immediately after surgery, followed by conventional adjuvant chemotherapy after radiotherapy, have also been conducted [17]. Unfortunately, neither of these approaches has shown clear evidence of a significant reduction in the recurrence rate, which would support the use of these techniques in place of conventional adjuvant chemotherapy to improve long-term outcome [18]. However, trials of short-duration chemotherapy given immediately after surgery have demonstrated that treatment in this period was effective; patients who received only postoperative perioperative chemotherapy and locoregional therapy in EORTC 10854 [17] had significantly better overall survival rates than patients who received locoregional therapy alone $(P=0.004)$. This suggests that effective therapy in the immediate postoperative period is unlikely to be harmful.

\section{The potential gain of prognostic and predictive information}

Breast cancer is a notoriously clinically heterogeneous disease. Categorisation based on gene expression classification using unsupervised analysis identifies five main subtypes, two of which contain two HER2 positive groups: 'HER2+ like' (ER-, HER2+) and ER+, HER2+ patients within the luminal B subgroup. However, even within these groups there is wide variability in outcome and treatment sensitivity. For example, after a median follow-up of 2 years in the HERA trial [11], 19\% of observation patients and $13 \%$ of trastuzumabtreated patients had experienced an event; thus targeted therapy had only reduced the event rate by approximately one-third. The use of preoperative treatment may offer the opportunity to obtain useful prognostic and predictive information that can be used as a basis for giving or withholding further treatment. If there is a relationship between the longterm outcome of patients and biological changes in the primary tumour in response to presurgical therapy, then these changes may accurately reflect the effect of therapy on micrometastases; however this hypothesis needs to be validated in controlled randomised trials. Biological changes in the blood, such as changes in angiogenic factors, may also offer useful information and their measurement can extend beyond the point of surgery.

An example of this phenomenon is provided by a biomarker substudy of the IMPACT study [19] (the substudy had a total of 25 events), which looked at the effect of biomarker changes induced by 2 weeks of treatment with tamoxifen and/or anastrazole in hormone receptor positive early breast cancer. Ki67 $(P=0.004)$ and ER scores $(P=0.04)$ measured after 2 weeks exposure to presurgical endocrine therapy were found to be superior prognostic factors to the same values measured before therapy. Tumour size measured at baseline was also found to be important, but it was not measured at 2 weeks when relatively little change would be expected. With regard to changes following anti-HER2 therapy, change in tumour size after 11 days of therapy may provide additional prognostic information that is not available from a pretreatment tumour specimen. Lapatinib has been reported [20] to shrink tumour size by $74 \%$ after 6 weeks which interpolates to a $36 \%$ shrinkage after 2 weeks. Moshin et al. [21] also found early 
tumour regression with a median decrease of $20 \%$ $(0-60.4 \%)$ after 3 weeks of trastuzumab; changes in apoptosis were also observed over this period. A study [22] of biomarker profiles that were predictive of response to lapatinib was undertaken in a phase II trial in 45 inflammatory breast cancer patients, out of which 30 were HER2 + . In these patients, those with phosphorylated HER-3 showed a higher response rate than those without this characteristic $(67 \%$ vs. $22 \% ; n=15$ and $11, P=0.02$ ) and lack of p53 was also predictive of response.

Gene expression prognostic indices are receiving increasing attention in cancer prognostication. The potential of gene expression indices based on changes in gene expression can be illustrated by a study undertaken by Mackay et al. [23], who undertook expression profiling using cDNA arrays from pretreatment and post treatment biopsies in 34 sample pairs from ER+ breast cancer patients treated with anastrozole or letrozole for 2 weeks. Significant changes in gene expression were observed, particularly oestrogen-dependent and proliferation-related genes. The authors explored this further by combining gene changes into an overall score, described as the Global Index of Dependence on Estrogen (GIDE), which summed the genes changing by at least twofold with therapy. GIDE was found to be related significantly to pretreatment levels of HER2 and changes in immunohistochemically detected Ki67.

An important characteristic of perioperative therapy is that it offers the opportunity to combine information from both pre-therapy and post therapy samples. The pre-therapy expression of a particular biomarker can be viewed as offering information on the inherent prognosis of the patient and the posttherapy expression offers information on the response to treatment. It is therefore preferable to treat these two values as independent factors initially and only combine them into a single prognostic factor if this can be shown to be appropriate. As a simple example, gene expression changes from one to three and from two to six both have a fold change of three; however a patient with the former fold change may have inherently different prognosis because the overall expression levels are lower. Although it is tempting to view biomarker changes as surrogate endpoints, it is important to remember that to identify a surrogate endpoint it is not enough to simply show that the biomarker level correlates with outcome. It must also be shown that changes in the biomarker correlate with changes in outcome [24].

A note of caution is appropriate about the assumption that new prognostic information will be available from the use of perioperative therapy. In NSABP-18 [25] prediction of outcome was no better in the presurgical chemotherapy arm than in the postoperative arm despite the fact that the former was able to incorporate factors such as pathological tumour response and pathological nodal response which reflected response to chemotherapy. However, scientific advances since NSABP-18 was conducted have resulted in more biological information now being obtainable and it is important to test whether this additional biological readout adds critically useful prognostic and predictive information.

\section{Evidence for the biological effect of anti-HER2 therapy}

Evidence for the effect of anti-HER2 therapy gained from the re-excision of tumours has been presented above; evidence is also available from a number of other sources. It has been suggested that stem cells are resistant to chemotherapy and are the origin of resistant disease. Organ-specific markers that are expressed by stem cells can be identified from solid tumours, for example, the cell surface marker profile, CD44+CD24-/lowLin- [26], was found to identify a tumourigenic stem-cell population in samples from eight of nine human breast cancers. This profile was found to be enriched upto 100 -fold with cells able to form tumours in mice. As expected of stem cells, the corresponding tumours in mice were found to possess similar phenotypic diversity to the original tumour population, encompassing subpopulations of both tumourigenic and non-tumourigenic cells. There is some evidence that anti-HER2 therapy, unlike chemotherapy, is effective against stem cells. Rodriguez et al. [20] carried out a presurgical study involving 45 patients which provided evidence suggesting that lapatinib decreases breast cancer stem cells in the primary breast cancers of women receiving presurgical lapatinib.

Short-term biomarker effects were also seen in a study by Spector et al. [14]. These authors studied the biological effects on selected tumour growth and survival pathways in patients with advanced ErbB1 and/or ErbB2-overexpressing solid malignancies after 21 days of different doses of lapatinib. Tumour response was assessed after 8 weeks and the series included 13 patients with advanced breast cancer, four of whom demonstrated a partial response. Despite the small number of breast cancer patients, evidence of a fall was shown in p-Erk1/2 $(P<0.01)$, TGF- $\alpha(P<0.05)$ and p-ERB1 $(P=0.03)$. There were also non-statistically significant falls in Cyclin $\mathrm{D}(P=0.065)$ and $\mathrm{p}$-Akt $(P=0.13)$ (Wilcoxon signed rank test, analyses performed by RA on data extracted from Tables 2 and 3 [14]). 
EGFR and HER2 may be involved in maintaining anueploidy in some cancers, which may therefore be responsive to anti-EGFR and anti-HER2 therapy. Cancer can be caused by aneuploidy (chromosomal gain, loss or rearrangement) and may not be attributable solely to gene mutations. Anueploidy was first suggested to play such a role in 1890 and is currently incorrectly commonly considered to be a late-stage effect rather than a cause of cancer development. Bjerkvig et al. [27], for example, have pointed out that carcinogens such as asbestos and arsenic initially produce aneuploid lesions and not genetic mutations and that normal cells exposed to chemical carcinogens can become aneuploid some time before they show signs of being cancerous. Mutations and chromosomal instability may therefore both be important in the early stages of tumour development and involved in genesis of the cancer stem cell. In ovarian cancer, the coexpression of EGFR and c-erbB-2 is 47-68\% [28]. Pack et al. examined the effects of antisense or immunosuppression of EGFR and c-erbB-2 expression on the invasive phenotype, aneuploidy and genotype of cultured human ovarian carcinoma cells. They found that the suppression of both EGFR and CerbB-2 resulted in a decrease in aneuploidy and genomic imbalances, and restored a more normal phenotype and a more normal gene expression profile. Other evidence also supported the fact that the regression of aneuploidy was due to the selective apoptosis of double antisense transfected cells with a highly abnormal karyotype. EGFR and HER2 may therefore play a role in maintaining anueploidy within cancers, a condition that may be countered by the use of anti-EGFR and anti-HER2 therapy.

\section{Conclusions}

This paper has contrasted the use of perioperative anti-HER2 therapy with the use of preoperative and perioperative cytotoxic chemotherapy, both of the latter have been tested in randomised clinical trials but have not been found to offer a benefit in terms of long-term outcome or enhanced prognostication. However, it is important to evaluate the role of perioperative anti-HER2 therapy because of its distinct modes of action, and scientific advances have resulted in the greater availability of biological information that can be used to study prognostic and predictive effects. Controlled trials of perioperative therapy present the best opportunity to investigate these characteristics. Perioperative antiHER2 therapy is of unproven benefit and safety, and it should therefore only be undertaken within the context of a randomised controlled trial such as EPHOS-B; however such a strategy requires both
HER2 status assessment at or close to diagnosis and cardiac assessment, requiring pathological services that can provide rapid diagnosis and the availability of timely ECHO or MUGA scans.

Questions relating to the EPHOS-B study can be addressed to the EPHOS Trial Coordinator within ICR-CTSU, Email: ephos-icrctsu@icr.ac.uk

\section{Acknowledgement}

The EPHOS-B trial (ISRCTN: ISRCTN63882543) is funded by Cancer Research UK.

\section{References}

1. Tagliabue E, Agresti R, Carcangiu ML, et al. Role of HER2 in wound-induced breast carcinoma proliferation. Lancet 2003; 362: 527-533.

2. Dowsett M. Preoperative models to evaluate endocrine strategies for breast cancer. Clin Can Res 2003; 9(Suppl): 502s-510s.

3. Bernard Fisher B, Bryant J, Wolmark N, et al. Effect of preoperative chemotherapy on the outcome of women with operable breast cancer. J Clin Oncol 1998; 16: 2672-2685.

4. Johnston S. UK Clinical Research Network: Portfolio Database (http://public.ukcrn.org.uk/search/), UKCRN Study ID: 2523, 2009.

5. Smith IE. UK Clinical Research Network: Portfolio Database (http://public.ukcrn.org.uk/search/), UKCRN Study ID: 4023, 2009.

6. Fisher B, Saffer E, Rudock C, Coyle J, Gunduz N. Effect of local or systemic treatment prior to primary tumor removal on the production and response to a serum growth-stimulating factor in mice. Cancer Res 1989; 49: 2002-2004.

7. Fisher B, Mamounas EP. Preoperative chemotherapy: a model for studying the biology and therapy of primary breast cancer. J Clin Oncol 1995; 13: 537-540.

8. Choy $A$, McCulloch P. Induction of tumour cell shedding into effluent venous blood breast cancer surgery. $\mathrm{Br} J$ Cancer 1996; 73: 79-82.

9. Liotta LA, Stetler-Stevenson WG. Tumor invasion and metastasis: an imbalance of positive and negative regulation. Cancer Res 1991; 51(Suppl 18): 5054s-5059s.

10. Ring A, Smith IE, Dowsett MC. Circulating tumour cells in breast cancer. Lancet Oncol 2004; 5: 79-88.

11. Smith I, Procter M, Gelber RD, et al. 2-year follow-up of trastuzumab after adjuvant chemotherapy in HER2positive breast cancer: a randomized controlled trial. Lancet 2007; 369: 29-36.

12. Joensuu $\mathrm{H}$, Kellokumpu-Lehtinen $\mathrm{PL}$, Bono $\mathrm{P}$, et al. Adjuvant docetaxel or vinorelbine with or without trastuzumab for breast cancer. N Engl J Med 2006; 354(8): 809-820.

13. Al-Hajj M, Wicha MS, Benito-Hernandez A, Morrison SJ, Clarke MF. Prospective identification of tumorigenic breast cancer cells. Proc Natl Acad Sci USA 2003; 100: 3983-3988. 
14. Spector NL, Xia W, Burris H, et al. Study of the biological effects of lapatinib, a reversible inhibitor of ErbB1 and ErbB2 tyrosine kinases, on tumour growth and survival pathways in patients with advanced malignancies. J Clin Oncol 2005; 23(11): 2502-2512.

15. Buzdar AU, Ibrahim NK, Francis D, et al. Significantly higher pathologic complete remission rate after neoadjuvant therapy with trastuzumab, paclitaxel, and epirubicin chemotherapy: results of a randomized trial in human epidermal growth factor receptor 2-positive operable breast cancer. J Clin Oncol 2005; 23: 3676-3685.

16. Perez EA, Suman VJ, Davidson N, et al. Advances in monoclonal therapy for breast cancer: further analysis of NCCTG N9831. 41st Annual Meeting of the American Society of Clinical Oncology, Orlando, FL, 16 May 2005.

17. van der Hage JA, van de Velde CJH, Julien J-P, et al. Improved survival after one course of perioperative chemotherapy in early breast cancer patients: long-term results from the European Organization for Research and Treatment of Cancer (EORTC) Trial 10854. Eur $J$ Cancer 2001; 37: 2184-2193.

18. Mieog JSD, van der Hage JA, van de Velde CJH. Preoperative Chemotherapy for Women with Operable Breast Cancer (Review). The Cochrane Collaboration: John Wiley \& Sons, Ltd, 2008.

19. Dowsett M, Smith IE, Ebbs SR, et al. Prognostic value of Ki67 expression after short-term presurgical endocrine therapy for primary breast cancer. J Natl Cancer Inst 2007; 99: 167-170.

20. Migliaccio I, Gutierrez MC, Wu M-F, et al. P13 Kinase activation and response to trastuzumab or lapatinib in
HER-2 overexpressing locally advanced breast cancer (LABC). Cancer Res 2009; 69(Suppl): 71s (abstr 34).

21. Mohsin SK, Weiss HL, Gutierrez C, et al. Neoadjuvant trastuzumab induces apoptosis in primary breast cancers. J Clin Oncol 2005; 23(11): 2460-2468.

22. Johnston S, Trudeau M, Kaufman B. Phase II study of predictive biomarker profiles for response targeting human epidermal growth factor receptor 2 (HER-2) in advanced inflammatory breast cancer with lapatinib monotherapy. J Clin Oncol 2008; 26: 1066-1072.

23. Mackay A, Urruticoechea A, Dixon JM, et al. Molecular response to aromatase inhibitor treatment in primary breast cancer. Breast Cancer Res 2007; 9: R37.

24. Collette L, Burzykowski T, Carroll KJ. Is prostatespecific antigen a valid surrogate end point for survival in hormonally treated patients with metastatic prostate cancer? Joint research of the European Organisation for Research and Treatment of Cancer, the Limburgs Universitair Centrum, and AstraZeneca Pharmaceuticals. J Clin Oncol 2005; 23: 6139-6148.

25. Fisher B, Bryant J, Wolmark N. Effect of preoperative chemotherapy on the outcome of women with operable breast cancer. J Clin Oncol 1998; 23: 6139-6148.

26. Dontu G, Al-Hajj M, Abdallah WM, Clarke MF, Wicha MS. Stem cells in normal breast development and breast cancer. Cell Prolif 2003; 36(Suppl 1): 59-72.

27. Bjerkvig R, Tysnes BB, Aboody KS, et al. The origin of the cancer stem cell: current controversies and new insights. Nature Rev Cancer 2005; 5: 899-903.

28. Pack SD, Alper OM, Stromberg $\mathrm{K}$, et al. Simultaneous suppression of epidermal growth factor receptor and c-erbB2 reverses aneuploidy and malignant phenotype of a human ovarian carcinoma cell line. Cancer Res 2004; 64: 789-794. 